\title{
Diagnóstico Situacional en un Centro de Capacitación para el Trabajo en México: Desafíos para Atender a la Diversidad
}

\author{
Situational Diagnostic into a Capacitation Work Center in \\ Mexico: Challenges to Address the Diversity
}

David U. López Quirino *

Juana $\mathrm{M}^{\mathrm{a}}$ Méndez Pineda

$\mathrm{M}^{\mathrm{a}}$ del Rosario Auces

Universidad Autónoma de San Luis Potosí

\begin{abstract}
En este documento se presentan los resultados de un diagnóstico realizado en un Centro de Capacitación para el Trabajo Industrial en la Ciudad de San Luis Potosí, México, que tuvo como objetivo conocer cómo se desarrollan las prácticas educativas para atender a una gran diversidad de estudiantes. Fue un estudio de tipo cualitativo en el que se realizaron observaciones participantes, análisis de documentos y entrevistas a docentes, alumnos y directivos. Luego de analizar los datos recabados, se concluyó que existen tres dificultades centrales en el centro: a) alumnado que abandona tempranamente las especialidades; b) dificultad de los docentes para dar atención a los grupos diversos; c) y la ausencia de un programa de orientación y tutoría institucional. Para las siguientes etapas, se implementará un programa de formación docente que integre un enfoque de educación inclusiva, estrategias del trabajo cooperativo, análisis reflexivo de la práctica docente y el desarrollo de proyectos, para incidir en dichas problemáticas.
\end{abstract}

Descriptores: Formación de docentes, Formación profesional, Programas de desarrollo, Práctica pedagógica, Tutoría.

This document presents the result of a diagnostic apply into a Capacitation of Industrial Work Center in the city San Luis Potosi, Mexico. It had the goal to investigate how are been improved the educational practices to attend the huge diversity of all the students. It was a qualitative study that included participative observations, the analysis of documents and interviews to teachers, students and directives. After of the analysis of the resorts, it was concluded that there are three focal difficulties into the Center: a) students leave too early the specialties; b) teachers difficulties to attend the different groups; c) and the fact that there isn't an orientation and tutorial program in the institution. For the next stage, it will be implemented a teacher training program that integrates an inclusive educational approach, cooperative work strategies, reflexive analysis of teacher's work and the development of projects, to contribute on that problems.

Keywords: Teacher education, Occupational training, Development programmes, Teaching practice, Tutoring.

*Contacto: david.lopez.quirino@gmail.com 


\section{Problemáticas de la educación en México: Los que se quedan en el camino}

El panorama actual de la educación para los jóvenes y su devenir laboral es una problemática que cada vez es más alarmante. Hoy en día esta población tiene necesidades laborales, sociales y culturales, a las que los gobiernos y sus economías no están dando respuesta. Tal como lo resalta el Fondo de las Naciones Unidas para la Población (2014) en su publicación más reciente sobre el estado de la población mundial, en el que refiere que un $60 \%$ de los jóvenes de entre 10 y 24 años a nivel mundial no estudia ni tampoco posee algún empleo, es decir, permanece en un estado de inactividad.

En lo que respecta al contexto mexicano, la Organización para la Cooperación y el Desarrollo Económicos (OCDE, 2013), ubica a México como el tercer país con mayor número de personas sin estar laborando ni estudiando (dentro de los 34 países que conforman esta organización), lo que representa el 24.7\% de los jóvenes de entre 15 y 29 años; esto es más de 7 millones de personas inactivas en la etapa más productiva de su vida.

Por tanto, la educación en nuestro país, y especialmente la de nivel medio superior, atraviesa por una época de grandes retos, principalmente por su carácter articulador entre la educación básica y la superior, es decir, es el lapso en el que se decide el rumbo que tomarán los jóvenes. Por su parte, la Encuesta Nacional de Deserción Escolar en el nivel Medio Superior (ENDEMS, 2012) reporta que por cada 100 alumnos que se inscriben al nivel primaria, únicamente 36 alcanzan a graduarse del nivel medio superior. Con ello podemos dimensionar la recurrente falta de oportunidades para ser educados, la pobre calidad de la enseñanza, así como la gran cantidad de personas que se quedan en el camino de la formación.

Por otro lado, el Instituto Nacional de Estadística y Geografía (INEGI, 2010) reporta que del total de la población de entre 15 y 19 años, el 43\% no asistió a la escuela, esto es, que una parte importante de la población en edades de formación en Nivel Medio Superior se encuentra ya laborando o en la inactividad. Por su parte la OCDE (2013), apunta que en México el 64\% de la población de entre 25 y 60 años, no alcanzó la formación en educación media superior. Lo cual remarca el reto de las instituciones y de quienes estudian a la juventud para incluir a estos sectores que quedan privados de la educación media superior y superior. Según el INEGI (2010) en San Luis Potosí, lugar dónde se desarrolló este trabajo de investigación, la escolaridad promedio de la población mayor de 15 años es de 8.3 años. En tanto, la media nacional es de 8.6 años; esto ubica al estado en el lugar 22 a nivel nacional.

Frente a esta situación, cabe preguntarse: ¿a dónde van los que no tienen actividad?, ¿de qué formas se está dando respuesta a estos retos por parte del sistema educativo?, ¿cómo participan los agentes educativos en ese proceso? y ¿qué pudiera estar llevando a los estudiantes al abandono? Estas son algunas de las cuestiones a las que se pretende dar luz con el presente estudio, a través del estudio realizado en un escenario educativo complejo, en el que resaltó el constante abandono de los estudiantes a sus especialidades, la falta de un programa de orientación educativa institucionalizado y la presencia de una amplia diversidad en el alumnado. 
Para cerrar este apartado, resaltaremos que es necesario concebir nuestra tarea en la educación desde una visión sistémica, histórica y dinámica, en la que todos los actores y elementos del contexto educativo, están relacionados entre sí y en constante comunicación. Con ello también queremos señalar que el contexto configura las relaciones y las prácticas educativas, tanto de forma positiva como negativa, por ello en este primer acercamiento a la institución, buscamos, ante todo, tratar de comprender y analizar su complejidad. No obstante, también habrá que asumir de manera crítica, la gran responsabilidad que la educación posee para nuestra realidad, en lo político, económico y social, pues como refiere Fernández-Enguita (2009) la educación en estos tiempos tiene una importancia para los países que nunca antes había tenido.

\subsection{Práctica de exclusión en las aulas: Relevancia de la inclusión y la colaboración}

Existen múltiples intervenciones para responder a situaciones problemáticas actuales como el abandono, la inactividad y la atención para todos, sin embargo, la propuesta de educación inclusiva, por ser una propuesta educativa y social, alcanza a dar respuesta de manera amplia a situaciones como éstas. Principalmente, la educación inclusiva propone un cambio de paradigma hacia la atención a la diversidad en las escuelas, transitando de un modelo deficitario, el cual parte desde la visión médica y asistencial; a un modelo competencial (López-Melero, 2004). Este último modelo plantea una visión más amplia de la labor educativa: una escuela para todas y todos sin exclusiones. Esta propuesta conlleva inicialmente una serie de cambios actitudinales y culturales para que la diversidad sea algo cercano y cotidiano.

En relación a la inclusión, también, consideramos importantes los fundamentos del Index for inclusion (Ainscow et al., 2000), en donde se propone el desarrollo de proyectos de mejora en centros educativos desde una visión inclusiva, contemplando los procesos y las relaciones que suceden dentro de las escuelas. Para entender más ampliamente lo que sucede dentro de las instituciones en relación a la inclusión, en el Index, se proponen tres dimensiones de análisis: "las políticas, las prácticas y la cultura de la institución" (p. 21). En esta propuesta también se considera que existen barreras para el aprendizaje y la participación, es decir, son las condiciones del contexto que pueden impedir el acceso al centro educativo o limitar la participación dentro de él.

Por su parte, López-Melero (2004) propone tres principios de esta nueva cultura: en primer lugar, el respeto, la tolerancia y la libertad de pensamiento para cambiar la práctica educativa y social; en segundo lugar, entender la diversidad como parte genuina y valiosa de la identidad de cada ser humano y, en tercer lugar, la importancia de vivir los valores dentro del contexto educativo, resaltando el amor como fundamento de la condición humana.

Por otro lado, Pujolás (2004) en pro de una adecuada atención a la diversidad, postula el aprender juntos a pesar de las características de cada estudiante, es decir, haciéndolo a la par y aprendiendo unos de otros (en comunidad y en colaboración). Por ello, este autor considera que no se puede educar separando a los alumnos normales de los diferentes pues algo así resulta incongruente con los valores de respeto, solidaridad y convivencia.

Al respecto Díaz-Barriga (2006) refiere que la simple proximidad física entre personas no define un grupo cooperativo, sino que éste se construye con las interacciones significativas entre sus integrantes. Así, en un grupo cooperativo las competencias sociales, el intercambio de ideas, el control de los impulsos, la diversidad y el diálogo se 
consideran importantes en esta forma de trabajo. Una manera sencilla de definir la cooperación es entenderla como el trabajar juntos para alcanzar metas compartidas, ejercicio que maximiza los aprendizajes de los integrantes de los grupos.

Las propuestas antes mencionadas, abren la perspectiva de lo que significa educar en la diversidad y en la colaboración, desde un enfoque crítico a las actuales prácticas educativas, las cuales hasta el momento se han quedado cortas en el tema de atención a la diversidad. Con estas visiones, se busca dar sustento del porqué la inclusión y la colaboración son alternativas actuales que pueden dar respuesta a las necesidades de cualquier contexto educativo, pues se persiguen condiciones de convivencia y participación abiertas, en donde caben todos los participantes de una comunidad, independientemente de sus características.

\subsection{La orientación y la tutoría inclusiva como alternativa a los entornos diversos}

Otro de los elementos relevante para éste trabajo es el de la Orientación y la Tutoría inclusivas como respuesta a las problemáticas del contexto actual. Se considera esencial que en una institución que prepara para el trabajo se cuente con actividades de esta índole para preparar mejor a sus estudiantes con el fin de acompañarlos en su proceso formativo.

Hoy en día existen algunos programas de orientación y tutoría de manera oficial en las escuelas públicas de nivel medio superior, como es el caso del Sistema Nacional de Tutorías Académicas el cual tiene como propósito "coadyuvar en la formación integral de los estudiantes atendiendo sus necesidades e interés, así como aquellos factores internos y externos que inciden de forma directa o indirecta en el proceso de aprendizaje y desempeño académico" (Subsecretaría de Educación Media Superior y Coordinación Sectorial de Desarrollo Académico, 2011, p. 16), sin embargo, estos programas no se implementan en todos los contextos como se esperaría.

Por otra parte, algunos autores como Bisquerra (2012) refieren que la orientación es un proceso de ayuda dirigida a todas las personas, cuyo objetivo es contribuir al desarrollo cognitivo, social emocional, moral, físico, etc. Para este autor, la orientación tiene la finalidad de formar personas para vivir y convivir en paz, en un entorno de democracia, construyendo conjuntamente el bienestar social y personal. Este tipo de aspectos, se vuelve relevante en nuestros contextos actuales, en dónde la tradición mecanicista del aprendizaje y los modelos tradicionales, nos exigen memorizar, saber hacer, dominar contenidos, etc., dejando de lado cuestiones medulares como la formación ciudadana, el pensamiento crítico y la convivencia social, aspectos más humanos y, desde nuestra perspectiva, los verdaderos fines de la escuela pública.

En este mismo rubro, Boza, Salas, Ipland, Aguaded, Fondón, Monescillo, y Méndez (2001), consideran que la orientación en el ámbito escolar, se concreta en tres elementos principales: educar es orientar para la vida, orientar es asesorar sobre opciones alternativas y orientar es capacitar para el propio aprendizaje. Por su parte, Santana y Álvarez (1996), proponen la orientación sociolaboral, ya que plantean que en la educación hay un exceso de enseñanza tradicionalista enfocada en la transmisión del saber, que deja de lado los aspectos socioculturales y económicos, así como las nuevas exigencias del mundo del trabajo.

Desde estas perspectivas en torno a la orientación es que el presente estudio pretende abonar para el desarrollo de nuevas prácticas educativas y el tránsito a escuelas más 
inclusivas, ya que, como postula Cisneros (2010): "es necesario ofrecer una atención educativa adecuada a todos y a todas, sin importar características físicas o intelectuales, situaciones culturales, religiosas, económicas, étnicas o lingüísticas" (p. 6).

\title{
1.3. La formación de docentes críticos de su propia práctica
}

En este apartado se resalta la importancia de que los profesores se formen más allá de la especialización y de la actualización, es decir, que los docentes tengan acceso a una formación centrada en el análisis de su propia práctica, a fin de que se cuestionen lo cotidiano y los supuestos que componen su forma de ser profesores. Esto implica convertirse en un agente crítico, reflexivo y transformador de sus contextos educativos como lo postula el enfoque Crítico Progresista. Al respecto, Barraza (2010) postula que los fines de dicho enfoque son que los profesores alcancen una práctica que articule la investigación con la intervención en un marco institucionalizado de profesionalización; el cual conduce a la autonomía profesional. El autor parte de los siguientes pilares:

\begin{abstract}
Primeramente, la teoría crítica de la ciencia social y de la educación en pro de una mejora educativa; en segundo lugar, la autonomía profesional y social de sus agentes; en tercer lugar, el diálogo y la colaboración como principales estrategias de desarrollo; y finalmente, la autonomía profesional como un proceso colectivo y emancipatorio que busca la transformación de las prácticas educativas. (p. 13)
\end{abstract}

Por su parte, Perrenoud (2004) postula que la práctica reflexiva de docencia debe concebirse como una postura permanente, es decir, como un proceso de entrenamiento inacabado, intensivo y voluntario. Dicho proceso se desarrolla en plena acción y sobre la acción docente, por ello el autor apoya la idea de convertir a los profesores en actores de su formación que acepten la incertidumbre, el riesgo y la complejidad, como formas de implicarse en su práctica.

En éste marco del análisis de la práctica, es donde encontramos un paradigma distinto desde el cual se pueden articular intervenciones de gran trascendencia en este contexto educativo, pues podemos entender la docencia como una función compleja y amplia, de la que nos podemos servir para alcanzar objetivos nuevos y más responsables con docentes más activos y transformadores.

\subsection{El qué y para qué de los CECATI}

Actualmente en México, los Centros de Formación para el Trabajo Industrial (CECATI) forman parte de la Subsecretaría de Educación Media Superior (SEMS), cuya misión es ofrecer alternativas de desarrollo educativo congruentes con el entorno económico, político, social, cultural y tecnológico de la nación (Dirección General de Centros de Formación para el Trabajo, 2013). El Nivel Medio Superior comprende bachillerato y preparatoria, así como la educación profesional que no requiere bachillerato o sus equivalentes; entre éstos últimos se encuentra la capacitación para el trabajo.

La capacitación para el trabajo está dirigida a atender a las personas con intenciones de formarse para el trabajo (Subsecretaria de Educación Media Superior, 2013b), lo que les confiere un estatus particular a las demás instituciones de su nivel. Entre sus particularidades, estos centros atienden poblaciones de diversas edades, personas con distintos perfiles académicos y cada capacitación es sustancialmente diferente a las demás. Ésta heterogeneidad de condiciones pone a los CECATIS en desigualdad de condiciones, pues no se empata con la forma de trabajo que predomina en el sistema. 
Los CECATI ofrecen cursos de capacitación para y en el trabajo. Estos centros capacitan a las personas bajo los principios de pertinencia, calidad y equidad, sin importar su escolaridad; actualiza y especializa los conocimientos y las habilidades técnicas de los trabajadores; valida las competencias adquiridas de manera práctica o por autoaprendizaje, y estimula en los estudiantes y egresados la creación de empresas (Dirección General de Centros de Formación para el Trabajo, 2013).

\subsection{1. ¿Quiénes conforman la comunidad educativa de este centro?}

El CECATI objeto de este estudio es un centro que tiene mucha flexibilidad y apertura para los alumnos (Cisneros, 2010), pues atiende a personas de 15 años en adelante, con mínimos requisitos de ingreso. Por ejemplo, no siempre se requiere grado de escolaridad o experiencia, ni cubrir colegiaturas costosas para ser admitido. Además, hay talleres que cuentan con cuatro horarios distintos para el mismo curso, con el fin de poder atender a personas que trabajan e incluso a los que rolan turnos.

Los alumnos que acuden a CECATI tienen características como la diversidad de edades, de formación académica, de intereses, de procedencias y de "capacidades", las cuales no se ven en la mayoría de instituciones educativas; aquí se trabaja y convive diariamente con la diversidad, lo que hace aún más relevante el estudio de este contexto. Además, este plantel está ubicado cerca de la zona industrial de la ciudad de San Luis Potosí, con la finalidad de captar personas ligadas con la industria.

Durante el ciclo 2013-2014 se inscribieron 2556 estudiantes en sus distintos talleres. La planta docente se compone por 18 profesores y cuenta con 20 trabajadores, entre directivos, administrativos y personal de limpieza. Este plantel ofrece formación diversa en más de 10 talleres como son el de máquinas y herramientas, inglés, informática, aula POETA, diseño y fabricación de muebles de madera, contabilidad, mecánica automotriz, estilismo y bienestar personal, electrónica, entre otros.

\subsubsection{El Aula POETA, un espacio para los "especiales"}

Una de las particularidades de este centro es la incorporación, hace aproximadamente seis años, del Programa de Oportunidades para el Empleo a través de la Tecnología en las Américas (POETA). El programa denominado Aula POETA fue creado por la organización Trust for the Américas en el año 2004, una Fundación de la Organización de Estados Americanos (OEA) que se encarga de capacitar a personas con "discapacidad" en el uso de Tecnologías de Información, proporcionando, a través de la educación, acceso a posibilidades de empleo y una participación social más activa (Subsecretaria de Educación Media Superior, 2013a).

Por su parte, el sistema CECATI plantea que el aula POETA no sólo capacita a los participantes en computación básica, sino que también ofrece el desarrollo de habilidades para desempeñarse mejor en el campo laboral. Por ejemplo, en cuestiones como la interacción social, la preparación para el trabajo, las relaciones con clientes, la resolución de conflictos, el liderazgo, el trabajo en equipo y autoestima. Además, se encontró que el Programa tiene 134 centros operando en 20 países de América incluido México, donde inició operaciones en el año 2005 en la Universidad Pedagógica de Campeche y, cuatro años más tarde, el Gobierno de México, en Alianza con Trust for the Americas y la empresa Microsoft, fortalecieron el programa con la creación de 45 Aulas POETA. Cabe mencionar que esta iniciativa fue apoyada por fondos del Congreso de la Unión a través de la Secretaría de Educación Pública (SEP). Según el directorio POETA (Subsecretaria de 
Educación Media Superior, 2013c), hay una red de 53 aulas repartidas en prácticamente todos los estados, ubicadas por lo general en los CECATI; cabe mencionar que dicha red de Aulas POETA, es la más grande de América Latina.

Cisneros (2010) refiere que antes del Aula POETA, en los CECATI ya se daba atención a las personas con "discapacidad", y presenta una estadística de las personas con esta condición atendidas del 2000 al 2008. En ésta se observa un incremento de inscripciones de las consideradas "personas con discapacidad"; de 1966 personas en el ciclo 2000-01 a 6600 en el ciclo 2007-08. Con este tipo de movimientos institucionales, se acerca a esta población con discapacidad a las aulas y al mundo del trabajo, brindándoles un espacio designado para su formación que no poseían con anterioridad.

Finalmente, señalamos, que según con lo revisado en torno a la red POETA en México, ésta representa un modelo bien adoptado por los CECATI, por su estructura organizativa, por su accesibilidad y alcance a nivel nacional, pues como refiere Cisneros (2010), la particularidad del CECATI, es la flexibilidad para el ingreso, cuotas accesibles, cursos de corta duración y el sentido práctico. Finalmente diremos, que a pesar de las intenciones y promesas que el programa POETA refiere, aún se desconocen los logros obtenidos a partir de su incorporación en el contexto mexicano, lo cual es un asunto pendiente por explorar.

\subsubsection{Justificación}

Se seleccionó al CECATI también por ser una institución de educación pública, que como ya se dijo, posee peculiaridades de interés en el ámbito educativo. Debido a la complejidad de las situaciones educativas del centro, creemos que un diagnóstico como éste puede ofrecer elementos para entender la configuración de la dinámica y los retos que se enfrentan profesores, estudiantes y autoridades en la emprenda de construir una escuela para todos.

Resulta relevante hacer ésta exploración, ya que estudios como éste pudieran dar luz para la mejora de la atención al alumnado y a la transformación del profesorado. Además, aspectos como la colaboración, la inclusión y la orientación en la escuela, son áreas que hasta el momento no se ha explorado en una institución de este tipo. Finalmente diremos que la educación en nuestro país atraviesa por momentos de transición a un nuevo modelo educativo. Y por ello, es nuestra responsabilidad como profesionales inmersos en la educación, interesarnos en investigar y tratar de entender la complejidad de nuestros contextos.

El objetivo general de este diagnóstico fue conocer las condiciones actuales del centro en cuanto a su cultura y sus prácticas para la atención a la diversidad, la colaboración y la orientación educativa, y analizar cómo se relacionan con el desarrollo de los docentes y alumnos. En cuanto a los objetivos específicos se plantearon los siguientes: a) Conocer la diversidad de estudiantes que tiene este centro; b) Explorar cómo es la atención que se brinda a los alumnos considerados "diversos"; c) Conocer las características del profesorado; d) Identificar los principales retos del centro; e) Conocer las experiencias en orientación educativa; d) Identificar las limitantes organizativas, físicas y socioculturales para la implementación de proyectos de mejora. 


\section{Método}

Este diagnóstico partió de un enfoque cualitativo (Barraza, 2010) para la elaboración de Propuestas de Intervención Educativa. De esta propuesta se retomó la indagación inicial de los contextos educativos, que consistió en la utilización de técnicas e instrumentos de tipo cualitativo y el acercamiento con los principales actores o informantes clave del centro. En este sentido consideramos importante el enfoque de la inclusión, ya que, desde su visión, la inclusión está relacionada con un exhaustivo análisis de todas las formas a través de las cuales los centros escolares pueden marginar o excluir al alumnado. Además, se considera que la inclusión de estudiantes mejora cuando la escuela trata de responder a todos los aspectos de su diversidad (Ainscow et al., 2000).

De esta propuesta para entender los centros educativos, se planteó una valoración inicial (diagnóstica) desde una visión más social, considerando las barreras para el aprendizaje y la participación que se enfrentan. Además, se propuso que, para explorar la inclusión en centros educativos, se debe realizar a partir de tres dimensiones, la cultura, las políticas y las prácticas, mismas que se consideraron en función de otras problemáticas para la conformación del presente estudio diagnóstico (Ainscow et al., 2000).

\subsection{Técnicas e instrumentos de recolección de datos}

Para recabar la información del centro se utilizaron técnicas de manera estratégica y sistemática, los instrumentos que se utilizaron previamente fueron aplicados en otros contextos para optimizarlos y validados por expertos en el área, es decir, los instrumentos fueron diseñados por el autor, se pilotearon con compañeros y personas dedicadas a la docencia, y en todo momento fueron revisados por los asesores del proyecto (docentes investigadores) dentro de un seminario de Investigación en Educación Inclusiva. A continuación, se describen los instrumentos utilizados para la recolección de datos, los cuales se desprenden de técnicas como la observación participante, la entrevista y el análisis documental.

Se utilizó la observación participante, desde un enfoque etnográfico (Bertely, 2001; CONAFE, 2006; Shagoury y Miller, 2000), la cual postula un acercamiento a las situaciones reales de los acontecimientos educativos, esto es, interactuando dentro del centro y dentro de las aulas. Para el registro de las observaciones se utilizó el diario de campo en donde se llevó un registro posterior por escrito de lo observado haciendo reflexiones personales.

En segundo lugar, ser recurrió a una guía de observación participante en el aula, mediante la cual, se hicieron anotaciones de aspectos específicos como a) el contexto, b) el ambiente, c) los participantes, d) comportamientos y actividades de los participantes, e) interacciones informales y actividades no planeadas, y finalmente a f) el lenguaje de los participantes (Barraza, 2010). Además, se recogieron documentos de la clase como ejercicios y prácticas

Se realizaron entrevistas semiestructuradas a partir de guías previamente elaboradas, las cuales fueron utilizadas para conocer la percepción de viva voz, de los principales actores del contexto educativo (informantes clave). Consideramos al igual que Mayorga (2004), que la entrevista es una técnica cualitativa que permite recoger una gran cantidad de información de una manera más cercana y directa entre investigador y sujeto de 
investigación. Se abordaron temas como la atención a la diversidad, la orientación en el centro, las relaciones que, entre los actores, los retos del centro, entre otros.

En cuarto y último lugar, mencionamos el análisis de documentos que consistió en recuperar información de documentos oficiales (Proyecto del Centro, Proyecto Curricular, Reglamentos, Organización del Centro) y documentos públicos (Revistas, Cartas, Comunicados). La finalidad fue reconstruir la propia realidad escolar vivida a través de los documentos.

\subsection{Procedimientos para el análisis de los datos}

Para realizar el análisis de los documentos se realizó una matriz de análisis documental, la cual consistió en una tabla de doble entrada, en la que se conjuntaron todos los documentos recabados con sus descripciones y reflexiones en torno a los temas de interés. Se pretendió elaborar, a partir de un documento bruto, un documento secundario que represente su contenido para facilitar su consulta o localización (Travé, 1996).

Respecto a las observaciones y entrevistas se hizo el análisis de los datos, de acuerdo con lo propuesto por Bertely (2001), quien sugiere sustraer la información y poder hacer interpretaciones para la construcción de los resultados a través de procedimientos como la triangulación teórica, la categorización y análisis de patrones emergentes. Además, consideramos lo referido por Shagoury y Miller (2000), quienes establecen la confección de índices, el método de comparación constante y la triangulación para analizar los datos.

Primeramente, las entrevistas, observaciones, diarios y demás datos recabados se recuperaron de manera textual para guardar la fidelidad de lo expresado por los informantes. Posteriormente se identificaron los temas generales en los datos recabados, con lo cual se empezó el proceso de categorización, es decir, se organizó la información encontrada en temas centrales (categorías). En este estudio se conformaron las siguientes categorías: a) Estado actual de la Orientación, b) Relaciones entre los actores, c) Características del docente, d) Características de los alumnos, e) Políticas de la institución.

Después se elaboró un índice con la ubicación de cada cita de cada categoría y se elaboró una matriz con las citas textuales, lo que facilitó la redacción. Para simplificar la información recabada, se construyó una relación de claves para identificar a los participantes, los instrumentos aplicados y los escenarios considerados durante el diagnóstico, dicha relación es presentada en la tabla 1.

Tabla 1. Relación de claves identificadoras para los elementos del diagnóstico

\begin{tabular}{llll}
\hline MARCA & \multicolumn{1}{c}{ SIGNIFICADO } & \multicolumn{1}{c}{ MARCA } & \multicolumn{1}{c}{ SIGNIFICADO } \\
\hline E- & Guía de Entrevista & IMA & Instructor Mecánica Automotriz \\
O- & Guía de Observación & ICr & Instructor de Carpintería \\
DI & Diario del Investigador & IAPo & Instructor Aula POETA \\
DOC & Documentos & IIn & Instructor de Inglés \\
TMA & Taller Mecánica Automotriz & AMA & Alumno Mecánica Automotriz \\
TCr & Taller Carpintería & ACr & Alumno Carpintería \\
TAPo & Aula POETA & AAPo & Alumno Aula POETA \\
TIn & Taller de Inglés & Ain & Alumno de Inglés \\
JCa & Jefa de Capacitación & JSA & Jefa de Servicios Administrativos \\
\hline
\end{tabular}


Posteriormente, se triangularon los datos entre instrumentos y también con la teoría, todo con la finalidad de tratar de comprender lo que sucede al interior de este plantel, como se describirá a continuación.

\section{Resultados}

En este proceso de conocer el estado actual de la institución, se pudo escuchar de viva voz la perspectiva y el sentir de los estudiantes, profesores y autoridades. A continuación, se desglosan los resultados del diagnóstico, esto a partir del análisis de los datos y con respecto a las triangulaciones con la teoría anteriormente señaladas y considerando la experiencia del facilitador. Estos resultados se presentan en tres grandes categorías de análisis: a) La institución habilitadora, la última oportunidad, b) Las otras diversidades: los que las otras escuelas ya no aceptaron y desechan, y c) Docentes empíricos, hacedores de personas útiles.

\subsection{La institución habilitadora, la última oportunidad}

"La institución habilitadora, la última oportunidad" es la primera categoría construida para dar cuenta de la situación educativa del papel del CECATI como institución y como es vista desde sus adentros. Iniciaremos retomando lo que se recogió mediante las entrevistas y observaciones, en donde profesores y autoridades afirmaron que el CECATI es una institución que educa a toda aquella persona que desea prepararse para la vida laboral, es decir, abre sus puertas de formación prácticamente a cualquier persona que busque un espacio en sus aulas. Decimos que es una institución habilitadora ya que de acuerdo con gran parte de los entrevistados (E-IIn1; E-AMA1; E-JSA1; E-IAPo3), el CECATI busca la preparación técnica de adultos y adolescentes inactivos profesional y laboralmente; quienes previo a estar inscritos han pasado un tiempo sin actividades, en otras instituciones de las cuales han sido dados de baja o han abandonado.

Igualmente se precisó que, en comparación con otros centros educativos, el proceso de ingreso, los reglamentos y los horarios generalmente son flexibles, otorgando libertad y espacios de desarrollo para el alumnado (E-AMA 2; E-IMA 2). Otra de las características representativas del centro es la prevalencia de la práctica dentro del currículum, "aquí la educación es más práctica que teórica, nosotros hacemos un $80 \%$ de práctica y $20 \%$ de teoría (...) estamos más enfocados a la industria, es por eso que los capacitamos más en la práctica” (E-Jcp 4). En este sentido también se obtuvo, que el modelo pedagógico que se sigue en las aulas, generalmente es técnico y tradicional, es decir, uno en el que se tienen programaciones y contenidos organizados por el docente, centrados en la transmisión del conocimiento, que carecen de participación y construcción por parte del alumnado, tal como se observó durante las observaciones de clases (OP-CIn; OP-CMA; OP-CAPo; OP-CCa), en las que generalmente el docente hablaba la mayor parte del tiempo y daban espacio para plantear dudas, mismas que raras veces se expresaban.

Aunado a ello, la institución posee una visión integradora de la diversidad, lo cual se ve reflejado en sus políticas, en la cultura y en sus prácticas, lo que ha limitado el desarrollo de sus alumnos (E-IAPo7; DI 6, 7 y 8). Aún se cuenta con espacios excluyentes, como es el caso del Aula POETA para personas con discapacidad, misma que se comenta más detenidamente en la siguiente categoría. No obstante, podemos deducir que el centro atraviesa por un proceso de transición a la inclusión debido a que cuenta con una 
infraestructura física adecuada (rampas, señalética apropiada, pasamanos, cajones reservados de estacionamiento, etc.) constatada en lo recabado en el diario del investigador (DI01), lo cual refleja la intención de la institución por ser inclusiva. Sin embargo, observó que, a pesar de este equipamiento, regularmente no se respetaron los espacios exclusivos de estacionamiento, el acceso al baño adaptado permanecía cerrado con llave y las reglamentaciones excluyen de certificación a esta población (DI03; DI05; E-Ica 5; E-IMA; E-ICr 9). Cuestiones como éstas, denotaron que aún no se cuenta con la cultura de una escuela inclusiva que visualice la diferencia como un valor (López-Melero, 2004).

Por otro lado, se encontró que el centro representa una última oportunidad de formación, sobre todo para los estudiantes que vienen de fracasos en otras escuelas y de la inactividad. Así lo expresaron los entrevistados, entre ellos el área directiva, que señaló que: "son alumnos que dejaron de estudiar o alumnos que salieron reprobados (...) o bien, son los que no encontraron preparatoria" (E-Jcp 6), por su parte el profesorado afirmó que son estudiantes que: "no terminaron secundaria porque no quisieron seguir estudiando, son gente que ya no busca una profesión” (IMA 5).

A pesar de que el CECATI se reconoce como una opción atractiva para esta población (EIMA 6), una parte importante de los estudiantes no logra concluir los cursos. Por un lado, la institución refirió que en el ciclo 2013-2014, tuvo un 81.8\% de eficiencia terminal, lo cual quiere decir que, de sus 2556 estudiantes inscritos, acreditaron los cursos 2091 (DOC-Estadística). Sin embargo, los profesores refirieron que ellos contemplan el abandono y la deserción bajo otros parámetros, pues consideran los cursos completos, esto es, tomando en cuenta el número de alumnos que inician una especialidad, contrastándolo con los que no logran certificarse por completo: "la deserción es uno de los principales problemas que tenemos porque realmente entran los grupos llenos y al final del curso son muy poquitos" (E-Jcp 6), refirió una de las profesoras.

Además, en este rubro, llama la atención que una institución como ésta no cuente con un programa de orientación educativa institucional, considerando que, en este proceso de formación para el trabajo, la orientación es de gran apoyo y promueve una mejora en el futuro del alumnado (Santana y Álvarez, 1996). Cabe señalar que a pesar de que no se retoma en este CECATI, existe el "Sistema Nacional de Tutorías Académicas para el Bachillerato General, Tecnológico y Profesional Técnico" (Subsecretaría de Educación Media Superior Coordinación Sectorial de Desarrollo Académico, 2011), creado por la misma Secretaría de Educación Pública para el Nivel Medio Superior con el fin de:

\begin{abstract}
“orientar la planeación, organización y operación de las tutorías académicas en los planteles (...) pretende apoyar a los alumnos en la resolución de problemas de tipo académico, coadyuvar en la promoción de su autonomía y formación integral, así como contribuir a mejorar su rendimiento académico (...) para que el estudiante desempeñe un papel más activo en el proceso enseñanza aprendizaje, promueva la creación y recreación del conocimiento y desarrolle habilidades, destrezas y actitudes, en el aspecto académico en pro de su permanencia en la educación media superior" (p. 8).
\end{abstract}

\title{
3.2. Las otras diversidades: los que las otras escuelas ya no aceptaron y desechan
}

En esta categoría sobre la población, resaltamos que a este centro acuden los denominado grupos desfavorecidos, ya sea por cuestiones económicas, escolares o sociales. Esto nos dimensiona el reto mayúsculo que enfrentan estos agentes educativos 
en general, ya que todos los días buscan educar en la diversidad, tratando de que ésta educación alcance a todos de manera equitativa. Por su parte, las autoridades y docentes refirieron una marcada clasificación entre tipos de alumnos (E-Jcp 6, E-IMA 6): los jóvenes, los adultos o mayores y las personas con discapacidad.

Primeramente, los jóvenes fueron descritos como el grupo más numeroso que generalmente lo componen menores de 18 años, los cuales en la mayoría de los casos no estudiaban ni trabajaban antes de incorporarse al centro (E-IMA 4; E-IAPo 7). Ellos fueron percibidos como los que "vienen más a jugar... a echar relajo", y que por algún motivo decidieron no seguir estudiando o no pudieron continuar. Además, han estado en los cursos en contra de su voluntad (E-IAPo 7; E-Jcp 6; E-ICr 5; E-IIn 4 y 5 ) y se reportaron como el grupo con mayor necesidad de orientación (E-ICr 7), atribuyendo este rasgo a que "son muy inmaduros, muy juguetones... un grupo muy difícil" (E-IIn 6). Lo observado en las aulas fue que se presta menos atención a este grupo e incluso fueron alumnos que abandonaban las aulas antes del final de la clase (OP-CI; OP-CM; OP-CC).

El segundo grupo lo conformaron los alumnos adultos o mayores, de los que se encontró de 1 a 5 por aula aproximadamente. A ellos se les refirió como "los más responsables, comprometidos, los que vienen a trabajar" (E-Jcp 6, E-IIn 5), "vienen a aprender, y realmente tienen vocación" (E-IIn 4). Ésta preferencia se pudo observar también en las aulas, pues evidentemente se dedicaba más tiempo y se prestaba mayor a atención a las personas de este grupo (OP-CI; OP-CM; OP-CC).

El tercero de los grupos, fue el de las personas con discapacidad, los cuales de acuerdo con los docentes también "son de trato difícil y riesgoso" (E-IAPo 9). Aquí se recogieron testimonios de renuencia, de descalificación y de segregación, sobre todo desde la óptica del profesorado. Comentaron que los alumnos con discapacidad llegan a un punto donde no pueden seguir por lo peligroso de las especialidades, en particular por el uso de los equipos (E-Jcp 9). Esta condición se vio expuesta, por las expresiones como: "el del año pasado no daba una el pobrecito" (E-ICr 9), "hay cierto rechazo de sus compañeros, se ríen de ellos... no les gusta sentarse con ellos... a veces veo que definitivamente no entienden" (E-IIn 7), "no le puedo decir, te garantizo que vas a aprender" (E-IIn 8). Todo esto, a pesar de que los alumnos con discapacidad suelen ser de los más constantes y con menores índices de deserción (E-IAPo 7; E-JCa 6). En general se les describe como estudiantes inferiores en capacidades y con bajas expectativas al egreso. Esto, aunado a una concepción de aceptación forzosa para el ingreso a sus grupos, y no como una cuestión inherente a su papel como docente. (E-IAPo 7; E-ICr 9; E-IIn 8). En este sentido, sostenemos que el papel del docente para superar las barreras de la exclusión es fundamental, ya que como refiere Fernández-Batanero (2013) el liderazgo de los profesores es un factor que puede determinar el avance o limitación de la inclusión.

Queda claro que, a pesar los esfuerzos por incluir a estos colectivos, las prácticas no han favorecido el proceso para la inclusión de todos. Lamentablemente esta clasificación del alumnado descarta a buena parte de ellos como sujetos de aprendizaje y los juzga como responsables totales de los procesos educativos fallidos, entre ellos el abandono escolar. Evidentemente estas prácticas de desentendimiento del docente, no mejoran la calidad de la enseñanza, sino que mejoran la comodidad del docente (Perrenoud, 2004), lo cual se reafirmó con expresiones como la siguiente: "somos el nivel educativo donde llegan los alumnos más desorientados... creo que nos llega lo peor o lo que todas las escuelas ya no aceptaron y desechan" (E-IMA 10). 
Finalmente se menciona lo que los alumnos refirieron en cuanto a sus propias dificultades, al respecto identificaron: la insuficiencia de prácticas profesionales (E-Jcp 10), la escasez de oportunidades laborales (E-AAPo10; E-AMA6) y las dificultades para ser independientes y autónomos (E-JSA 2, E-ICr 8 y E-JSA 4). Se resalta que desde el alumnado se reconoció una falta de responsabilidad, interés y compromiso en la propia formación (E-IIn 8).

\subsection{Docentes empíricos, hacedores de personas útiles}

En este centro, los docentes poseen peculiaridades por su diversidad de formación, áreas de adscripción, concepción de sus funciones, así como procederes, lo cual complejiza su identidad y participación dentro de la institución.

En cuanto a la comunicación entre los docentes, el estudio arrojó que han tenido pocas oportunidades de colaborar (E-IMA 2), así como escasa apertura para dialogar (E-ICr 9). Por ello refirieron sentirse faltos de respaldo y no poder trabajar en equipo, resaltando testimonios como: "soy un poco renuente a dialogar y me toca la suerte, de que yo solo me tengo que defender" (E-ICr 3), "considero que el clima es... muy frio, no hay compañerismo, no es de que yo veo que estás batallando, voy y te hecho la mano... te dejan morir solo" (E-IMA 2).

Algunos profesores expresaron rechazo a la innovación y una visión de su participación en el centro diferente a la de los más jóvenes: "somos maestros que ya deberíamos estarnos jubilando y ya no queremos cambios, no queremos conflictos... gente joven que empieza de todo quieren hacer pleito" (E-ICr 2).

Por otro lado, como ya se dijo, entre los docentes predomina un modelo tradicional y técnico de enseñanza, centrado en la transmisión del conocimiento (E-AMA 2), propiciando prácticas en las que se excluye a buena parte del grupo, ignorando lo que los alumnos pueden aportar (O-TMA 2). "La mayoría de los maestros hemos sido empíricos" (E-ICr 4), refirió uno de los docentes en torno a la docencia. En este mismo sentido, se reconoció que el objetivo del profesor se limita a instruirlos "para hacerlos personas útiles" (E-IMA 6 y 7). Lo dicho, coincidió con lo observado en las aulas, pues todos los docentes, se involucraron a diferente nivel en las actividades con los alumnos, con marcados favoritismos (O-TCr 3, O-TMA 3, O-TAPo 2 y O-TIn 4).

En gran medida, las clases que aquí se imparten, han obedecido la premisa ya mencionada de otorgar un $20 \%$ a la teoría y un $80 \%$ a la práctica, sin embargo, regularmente al momento de la práctica las clases se tornaron individualistas, selectivas, con poco trabajo en equipo, con tiempos muertos y como fue de esperarse, con recurrente ausentismo del alumnado (OP-Cr 1 y 2; OP-MA 1 y 3; E-IIn 1; OP-In 1).

Por otro lado, los profesores identificaron como retos centrales de su contexto: el abandono escolar (E-Jcp 6; E-IAPo; E-IMA 3), la dificultad de atender a grupos con diversidad (E-IMA 5; E-AI 4), la falta de formación constante por parte del centro en temas de docencia (E-IIn 3, 4 y 5; E-ICr 3 y 10; E- IAPo 2: E-IAPo 6 y 7 ) y la falta de material para ejercer su trabajo (E-IMA2).

Finalmente, se recabaron propuestas de mejora por parte de los profesores, las cuales se relacionaron con temáticas como la orientación del alumnado. Por un lado, se expresó la necesidad de que existiera dentro de la institución, una persona encargada de atención psicológica para los alumnos (E-IIn 9; E-ICr 8), con lo cual se denotó que las 
condiciones de los alumnos muchas veces también son emocionales, por lo que la función tutora del profesorado, se justifica ampliamente. Por otro lado, se propuso que se hiciera una evaluación, o una valoración sobre qué futuro se busca para el alumnado, de acuerdo a las necesidades del contexto (E-IMA 3).

\section{Conclusiones: Prioridades de mejora}

En síntesis, los resultados que arrojó el diagnóstico fueron: que la institución atiende a una diversidad importante de personas, que en ese momento no se contaba con un programa de orientación y tutorías institucional a pesar de su evidente urgencia y, finalmente que existía una percepción del abandono como un problema grave. Sostenemos que dichas temáticas estaban profundamente relacionadas entre sí, por lo que el impacto en cualquiera de ellas posibilitaría la mejora de las otras. Además, señalamos que, en las temáticas de atención a la diversidad, orientación educativa y abandono escolar, la inclusión representa un común denominador de éstas, ya que la inclusión pudiera dar respuesta a las problemáticas de esta institución por su sentido transformador de los contextos, lo cual atañe lo social y en lo pedagógico.

Además, con base en los resultados del diagnóstico, se consideró que la institución requería abordar sus problemáticas desde sus propios actores, específicamente, desde el personal docente; esto debido a que ellos son quienes hasta la fecha poseen mayor presencia en la institución, tanto en tiempo como en participación. Esto, a diferencia de los estudiantes lo cuales, debido a las características del plan de estudios y a las metodologías del CECATI, tienen poca permanencia y participación, aspecto que se agrava por el constante abandono de los mismos.

Por otro lado, la concepción actual de los profesores sobre sí mismos, limita también el campo de acción; pues los profesores no eran conscientes de su injerencia y protagonismo en su contexto, y se quedaban esperando a que la solución viniera desde afuera o desde arriba, específicamente de sus autoridades. Este diagnóstico nos mostró que los actores centrales de la institución, la mantenían estancada y en constante tensión; parecía que había mucho qué decirse los unos a los otros, pero no existían mecanismos o espacios para la comunicación, por lo que podemos asegurar que aún no existían condiciones para la mejora, ni para trabajar juntos. Por ello, sería necesario estructurar una propuesta que ayudara a la reconstrucción del centro, pero desde dentro, desde sus actores.

Por otra parte, mientras se siga partiendo de creencias como que no todos los estudiantes poseen igualdad de oportunidades, que existen buenos estudiantes y malos estudiantes; aptos y no aptos; deseables e indeseables, etc., la diversidad en las aulas seguirá siendo un problema para la práctica educativa, lo cual también seguirá propiciando que los estudiantes vayan de un lugar a otro sin encontrar su sitio, desaprovechando las oportunidades de formación que brinda el CECATI y a su vez desaprovechando las virtudes que la diversidad en la escuela tiene para dar.

A manera de cierre, se quiere enfatizar la importancia de atender las problemáticas más urgentes de los centros educativos, en éste caso del CECATI, sin dejar de lado la voz y la perspectiva de los mismos actores, así como sin minimizar su capacidad de acción conjunta. Esto nos resulta fundamental, siempre que se busque la mejora, ya que solamente cuando el centro se abra a la participación y se conciba como un colectivo con 
metas en común, podremos hablar de verdaderas soluciones de fondo; con acciones en las que participan todos los involucrados y se movilicen todos los recursos disponibles para enfrentar las problemáticas, mismas condiciones que nos puede proveer la educación inclusiva y la colaboración.

\section{Referencias}

Ainscow, M., Black-Hawkins, K., Booth, T., Goodey, C., Howkins, J., Jackson-Dooley, B., ... y Vaughan, M. (2000). Guía para la Evaluación y mejora de la educación inclusiva. Desarrollando el aprendizaje y la participación en los centros educativos. Londres: CSIE.

Barraza, A. (2010). Elaboración de propuestas de intervención educativa. Ciudad de México: Ed. Universidad Pedagógica de Durango.

Bertely, M. (2001). Conociendo nuestras escuelas. Un acercamiento etnográfico a la cultura escolar. Ciudad de México: Paidós Mexicana.

Bisquerra, R. (2012). Orientación, tutoría y educación emocional. Madrid: Síntesis.

Boza, A., Salas, M., Ipland, J., Aguaded, M., Fondón, M., Monescillo, M. y Méndez, J. (2001). Ser profesor, ser tutor. Orientación educativa para docentes. Huelva: Hergué.

Cisneros, B. (2010). Experiencias sobre la educación de aulas POETA en CECATI. Recuperado de http://www.educoas.org/portal/laeducacion2010

CONAFE. (2006). Modalidad de atención educativa a población indígena. El diario de campo. Ciudad de México: Consejo Nacional de Fomento Educativo

Dirección General de Centros de Formación para el Trabajo. (2013). ¿Quiénes somos? Conócenos. Recuperado de http://www.dgcft.sems.gob.mx/quienesomos.php?idcont=menu_pre111

Díaz Barriga, F. (2006) Enseñanza situada. Vínculo entre la escuela y la vida. México: McGraw Hill.

ENDEMS. (2012) Reporte de la encuesta nacional de deserción en la educación media superior, méxico. Recuperado de http://www.sems.gob.mx/

Fernández-Batanero, J. (2013). Competencias docentes y educación inclusiva. Revista Electrónica de Investigación Educativa, 15(2), 82-99.

Fernández-Enguita, M. (2009). Educar en tiempos inciertos. Madrid: Morata.

Fondo de las Naciones Unidas para la Población. (2014). Estado de la población mundial 2014. El poder de 1800 millones. Los adolescentes, los jóvenes la transformación del mundo. Recuperado de http://www.unfpa.org/

INEGI. (2010). Cuéntame. Población. Educación. Recuperado de http://cuentame.inegi.org.mx/poblacion/menu_edu.aspx?tema=P

López-Melero, M. (2004). Construyendo una escuela sin exclusiones. Una forma de trabajar en el aula Málaga: Aljibe.

Mayorga, M. J. (2004). La entrevista cualitativa como técnica de la evaluación de la docencia universitaria. RELIEVE, 1O(1), 23-39.

OCDE. (2013). Informe Panorama de la educación 2013 de la OCDE. París: OCDE.

Perrenoud, P. (2004). Desarrollar la práctica reflexiva en el oficio de enseñar. Profesionalización y razón pedagógica. Barcelona: Graó. 
Pujolás, P. (2004). Aprender juntos alumnos diferentes. El equipo de aprendizaje cooperativo en el aula. Barcelona: Eumo-Octaedro.

Santana, L. y Álvarez, P. (1996). Orientación y educación sociolaboral. Una propuesta curricular. Madrid: EOS.

Subsecretaría de Educación Media Superior Coordinación Sectorial de Desarrollo Académico. (2011). Sistema Nacional de Tutorías Académicas para el Bachillerato General, Tecnológico y Profesional Técnico. Ciudad de México: SEP.

SEP. (2012). Reporte de la encuesta nacional de deserción escolar en la educación media superior. Ciudad de México: México.

Shagoury, R. y Miller B. (2000). El arte de la indagación en el aula. Manual para docentesinvestigadores. Barcelona: Gedisa.

Subsecretaria de Educación Media Superior. (2013a). Programa de oportunidades para el empleo a través de la tecnología en las Américas. Recuperado de http://www.sems.gob.mx/es/sems/programa_oportunidades_empleo_poeta

Subsecretaria de Educación Media Superior. (2013b). ¿Qué es el SEMS? Recuperado de http://www.sems.gob.mx/es_mx/sems/que_es_la_sems

Subsecretaria de Educación Media Superior. (2013c). Ubicación de las aulas Poeta. Recuperado de http://www.sems.gob.mx/es/sems/ubicacion_aulas_poeta

Travé, G. (1996). Consideraciones sobre la utilización de técnicas e instrumentos de investigación educativa para la evaluación de unidades didácticas de contenido social. Revista de Investigación en la Escuela, 30, 87-97.

\section{Breve CV de los autores}

\section{David Uriel López Quirino}

Es Licenciado en Psicología por la Facultad de Psicología de la Universidad Autónoma de San Luis Potosí y actualmente cursa una Maestría en Psicología en la línea de formación "Psicología, Educación y Sociedad" en la misma universidad. Su trabajo e investigación aborda los temas de formación de profesores, atención a la diversidad e inclusión. Ha tenido participaciones en congresos nacionales e internacionales de educación, psicología educativa y de atención diversidad. Además, colabora en el Seminario Permanente de Investigación en Educación Inclusiva del Centro de Investigación, Orientación y Apoyo a la Inclusión en el Instituto de Ciencias Educativas de la Universidad Autónoma de San Luis Potosí. ORCID ID: 0000-0002-0894-5725. Email: david.lopez.quirino@gmail.com

\section{Juana María Méndez Pineda}

Actualmente es Profesora Investigadora de Tiempo Completo Nivel VI dentro de la Facultad de Psicología de la Universidad Autónoma de San Luis Potosí, catalogada con Perfil PROMEP 2013-2016 e Investigador Nacional Nivel 1 (SNI) 2013-2018. Imparte cursos en el nivel posgrado y licenciatura dentro de la misma universidad. Obtuvo el grado de Doctora en Educación en la Universidad Iberoamericana Plantel Golfo Centro, Las líneas de investigación en las que participa son Escuela y diversidad y 
Formación docente. Es Fundadora del Centro de Investigación Orientación y Apoyo a la Inclusión del Instituto de Ciencias Educativas de la Universidad Autónoma de San Luis Potosí, el cual desarrolla diferentes trabajos en investigación educativa en torno a la diversidad. También ha publicado libros de su autoría y en colaboración con otros autores, así como artículos en revistas científicas. Forma parte del cuerpo académico Escuela y Diversidad que conforma con otros investigadores afines. ORCID ID: 00000003-1851-976x. Email: jpineda@psicologia.uaslp.mx

\section{María del Rosario Auces Flores}

Es doctora en Educación con Campo en Formación de Docentes y Práctica Profesional por la Universidad Pedagógica Nacional Unidades Zacatecas - Ajusco. Las principales líneas de investigación que trabaja son: Escuela y diversidad, así como Formación docente. Profesora investigadora de tiempo completo Nivel VI dentro de la Facultad de Psicología de la Universidad Autónoma de San Luis Potosí, por lo que imparte cátedra en el nivel posgrado y licenciatura. Además, es coordinadora del programa de Maestría en Educación de la misma universidad, el cual se realiza en coordinación con el Instituto de Ciencias Educativas. También ha publicado libros de su autoría y en colaboración con otros autores, así como artículos en revistas científicas. Forma parte del cuerpo académico Escuela y Diversidad que conforma con otros investigadores afines. ORCID ID: 0000-0002-3785-4822. Email: charo_auces@hotmail.com 